\title{
A System for Energy Savings in an Ambient Intelligence Environment ${ }^{1}$
}

\author{
Thanos G. Stavropoulos ${ }^{1,2}$, Dimitris Vrakas ${ }^{1,2}$ \\ Alexandros Arvanitidis ${ }^{1}$, and Ioannis Vlahavas ${ }^{1,2}$ \\ ${ }^{1}$ Department of Informatics, Aristotle University of Thessaloniki, \\ AUTH campus, 54124 Thessaloniki, Greece \\ ${ }^{2}$ School of Science and Technology, International Hellenic University, \\ 14th km Thessaloniki - Moudania, 67001 Thermi, Greece \\ \{athstavr,dvrakas, arvanian, vlahavas\}@csd.auth.gr
}

\begin{abstract}
This work presents an Ambient Intelligence system that targets energy consumption awareness and savings. The system was deployed at the School of Science and Technology of the International Hellenic University and follows a three-layer approach. The first layer hosts devices (currently smart plugs, sensor boards and smart clampers) suited for the purpose. The second layer, namely the aWESoME middleware (a WEb Service MiddlewarE), resolves interoperability issues on the first layer, by universally exposing all actuator functions and sensor data through Web Services. Finally, a prototype application, named iDEALISM, has been developed to reside on the topmost layer. iDEALISM presents and manages all heterogeneous devices in the same place, enabling users to make comparisons, and take informed decisions on saving energy.
\end{abstract}

Keywords: wireless sensor networks, ambient intelligence, middleware, energy efficiency

\section{Introduction}

Ambient Intelligence (AmI) and ubiquitous computing are among the dominating technological paradigms of the near future. Mark Weiser has foretold this tendency: users surrounded by computing devices in the so-called pervasive or ubiquitous computing [1]. Indeed, nowadays, objects with increasing computing capabilities are penetrating the market and everyday life: Smartphones, internet-browsing and streaming-enabled TVs and motion detecting gaming controllers.

Smart devices have also widely spread due to affordability and the arising awareness for the environmental change. Many protocols, wired or wireless, have been designed to balance energy-efficiency and fulfill device networking requirements of Smart Spaces in small scale (i.e. smart homes, smart offices etc.) or large scale (i.e. smart buildings, smart cities). Domains of application range from home to health applications, government or agriculture.

${ }^{1}$ This project is funded by Operational Program Education and Lifelong Learning, OPS 200056 (International Hellenic University, Thessaloniki, Greece) 
A popular approach for AmI applications is the Service Oriented Architecture (SOA) in general, and, in some cases, a Web Service middleware. Web Services can expose data and functions in a universal way, forming the so-called Internet of Things.

This work presents an AmI application that aims, initially, at energy awareness and consumption reduction in the smart building domain. It was designed for a University, so it is suited for large scale distributed set-up, but also for small scale, e.g. smart homes. Architecture and fundamental ideas were previously presented in [2]. This work contributes the complete development of a middleware and a client application (aWESoME and iDEALISM respectively). The novelty resides in the fact that it targets energy savings in real life and large scale. Also, it builds upon ideas never applied to this domain, as reviewed in the next section.

The next section reviews related work and performs a short comparative analysis. The third section briefly reviews relevant hardware and presents the bottom layer of our system, which is a network of smart devices and sensors. The fourth section presents the middle layer of our system, e.g. the aWESoME middleware, while the fifth section presents the iDEALISM application on the topmost layer. The final two sections list future efforts and conclude our work.

\section{Related Work}

Home AmI applications either concern multimedia and/or home automation, as shown on Table 1. Otherwise, the system employs abstract/generic services. Multimedia applications always employ UPnP/DLNA services to stream media from and to various dynamically changing endpoints. However, that is irrelevant to our work, as energy savings can hardly profit from home entertainment. The most relevant of works, from this aspect, are home automation systems [9] and [11]. Home SOA [9], unifies multimedia services like UPnP, Bonjour etc. with home automation protocols like ZigBee [12] and X10 [13], by building drivers for them. However, there is no strategy to save energy, unlike in our work.

The Hydra middleware [11] is an FP7 project (soon to be renamed to LinkSmart) which also unifies heterogeneous home automation devices, by forming a Web Service Middleware. Our work differs in that Hydra doesn't propose some specific energy saving strategy. It also doesn't rely on composition to provide intelligent behavior. We specifically propose an application that exploits our middleware and presents consumption side by side with environmental data to raise awareness and enable informed decisions that save energy. We also plan to employ intelligent service composition in the future to improve results.

Most of the systems form a middleware. The rest of them do not or at least do not mention so. Moreover, some of the systems rely on Web Services and some not. Indeed, Web Services are sometimes redundant, like in the case of multimedia and UPnP. For example Interplay [8] composes UPnP actions-called 'Tasks', instead of Web Services, to obtain complex Tasks. Some of the systems attach semantic annotations to Web Services. This is a popular approach to render descriptions machine-interpretable and benefit from reasoning, automatic service discovery and matching. Finally, almost all systems employ intelligent service/task composition to provide users with complex functions in a hassle-free, automatic way. 
Table 1. Related work comparative table

\begin{tabular}{lccccc}
\hline System & Domain & Middleware & W. Services & Semantic & Composition \\
\hline [3] MEDUSA & - & Yes & Yes & Yes & Yes \\
[4] Mokhtar et al. & - & Yes & Yes & Yes & Yes \\
[5] Paluska et al. & - & No & No & No & Yes \\
[6] ResCo & - & Yes & No & No & Yes \\
[7] Kalofonos et al. & Multimedia & Yes & Yes & Yes & Yes \\
[8] InterPlay & Multimedia & Yes & No & No & Yes \\
[9] Home SOA & Mult./Autom. & No & No & No & Yes \\
[10] Mingkhwan et al. & Multimedia & Yes & Yes & Yes & Yes \\
[11] Hydra Middleware & Automation & Yes & Yes & Yes & No \\
\hline
\end{tabular}

\section{Smart Device Network}

Devices encountered in modern home automation can be classified as wired or wireless. Wired ones make use of power line cables (PLC technology) and belong mainly to the X10 family or its successor, the INSTEON[14] family. INSTEON devices are basically smart power consumption meters or controllers (switches) that are also backwards compatible with X10 devices. Like all PLC products, they utilize existent power cabling. Advantages of INSTEON, are router-free installation, interoperability of wireless and wired INSTEON nodes, unlimited number of nodes in a single network and highest reliability. However, INSTEON offers limited programmability and limited variety of devices (e.g. sensors).

Wireless products belong mainly to the ZigBee or the Z-Wave[15] family and are widely used in research and actual homes. Advantages include energy saving operation (thus, long battery life), forming of self-organizing mesh networks and adequate range. Their most important asset is the vast variety of devices, including environmental sensors (e.g. $\mathrm{CO}_{2}$ meters), smart power meters, motion detectors, and actuators like motors for doors or windows, TV and STB controllers, light dimmers and more.

Other devices used in ambient applications are RFID tags and cameras. RFID tags can be utilized to identify or locate persons or objects. Cameras together with Computer Vision algorithms can detect motion, identify persons or even classify their mood. Both trigger reactive automations.

Finally, wireless devices have been selected for our current implementation. Selecting incompatible products or adding more in the future is not an issue, given that the system's middleware provides the desired interoperability. Devices chosen can be classified as: Smart Plugs, Sensor Boards and Smart Clampers. They indeed belong to different manufacturers and do not interoperate out-of-the-box.

Smart Plugs. Smart Plugs can be regarded as sensors, as they measure the power consumption of appliances attached to them, and simultaneously as actuators, as they can switch the power supply of these devices on or off. Each plug is normally placed in a socket intersecting between an appliance and the socket. Then, that appliance can be switched on or off and its power consumption can be read. For any non-pluggable appliances (e.g. ceiling lamps or air conditioning), there are versions of the plugs that intersect cables (a fairly simple procedure). 
We chose a ZigBee bundle of around sixty Smart Plugs by Plugwise[16]. They form a dynamic mesh network of up to around thirty nodes. We chose to experimentally form three networks. Each one can be managed from a single PC equipped with a USB stick. As a bonus, some wall switches were also bundled, so users can control the devices in a traditional way. That improves overall user comfort and eliminates the constant need for a PC.

Sensor Boards. To broaden the variety of data, the system should support the use of different sensors, environmental or not. Again, many kinds of sensors are available in the market. In contrast to Smart Plugs, sensors are not a current trend but have been of interest long ago.

Requirements are however, that the sensors can form networks and are energy efficient themselves. We chose a sensor board platform by Prisma Electronics[17]. The platform consists of a ZigBee gateway that currently supports four sensor boards. The sensor boards operate in a long range, consume fairly a few energy and measure Luminance, Humidity and Temperature. More sensors can possibly be attached to the sensor boards.

Sensor data is collected by the Gateway and can be accessed over Wi-Fi, on a PC application. As a bonus, the code of the client application as well as a debugging kit for the boards comes with the bundle. That helps low-level configuring of the boards e.g. for sampling frequency, and greatly facilitates embodying the sensors in the middleware.

Smart Clampers. Up to this point, the system lacks large scale measurements of the power consumption. Smart Plugs can control appliances, in addition to measuring, but introduce a huge hassle and cost to connect every single power-draining appliance in the building. Even in the much simpler case of a home, using less than twenty appliances would probably result in leaving out appliances of critical consumption.

The solution to this problem is fairly simple and low-cost and involves installing one or more Smart Clampers. They simply clip around the main power supply cables of a building or apartment and, using an RF transmitter, send the data to a compact monitor and/or a PC application. The monitor offers an added, friendly interface and eliminates the constant need for a PC. As a result, no appliance is left unmeasured. In the case of a single household, a single 3-phase or 1-phase clip suffices.

We connected three 3-phase smart clampers by CurrentCost [19] plus their transmitters on the buildings power supplies. One of them corresponds to the university's data center and the other two correspond to the rest of the building. We also chose to support and test a set of three 3-phase OWL transmitters [18], and fully integrated them too. This process is described in the next section.

\section{The aWESoME Web Service Middleware}

aWESoME (a WEb Service MiddlewarE) has been developed for two purposes: to lift limitations on the first layer (devices) and to serve as a basis for AmI research, web or desktop applications. As they became apparent in the previous section, the most restraining limitations of devices are client PC placement, limited number of nodes per network, heterogeneity and incompatibility (e.g. a device only works in 32-bit OS etc). 
aWESoME resolves all issues as it allows any client placement, unifies networks, and integrates all data and platforms. As a result, any number of PC or microcomputer Gateways, Web Servers of aWESoME, can be assigned to serve devices within its range as shown on Figure 1. Application Logic (even Web Service Composition) can reside on local nodes (an IHU Agent, smartphone etc) or remote nodes via the Internet.

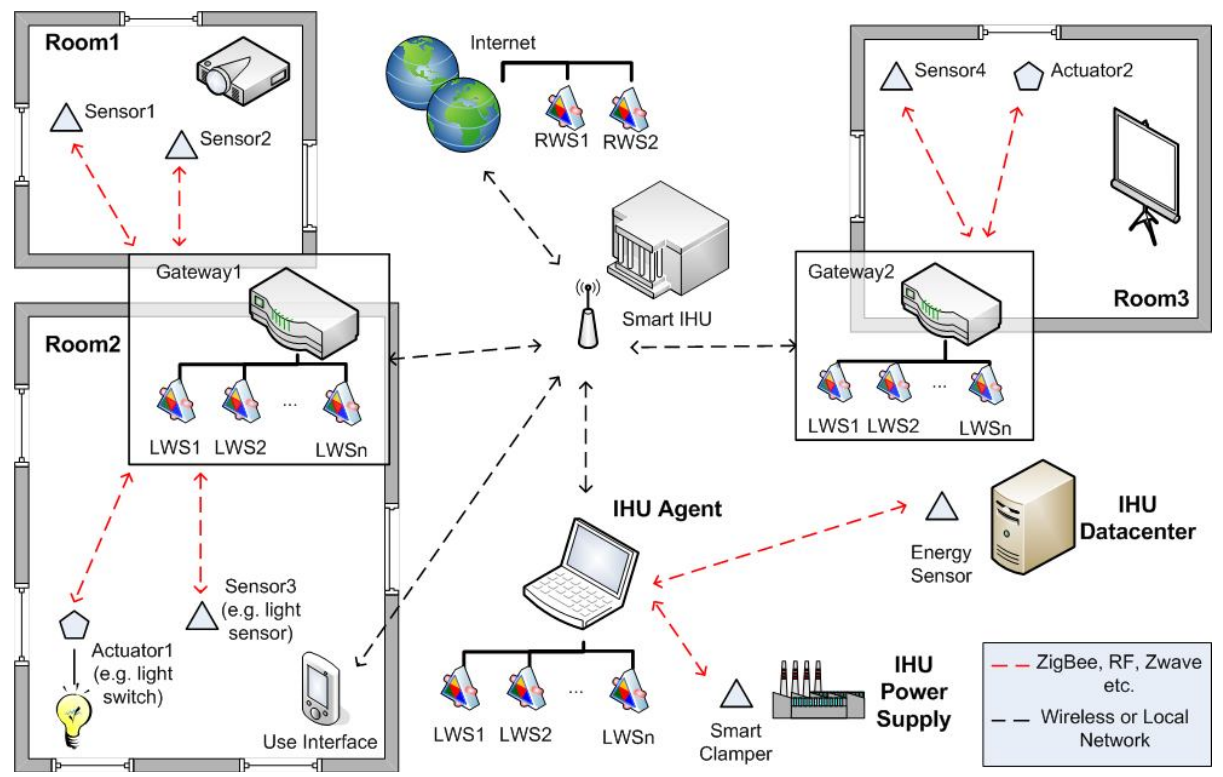

Fig. 1. Abstract aWESoME topology, adapted from [2]

Smart Plugs. Smart Plugs are sensors and actuators at the same time. Requirements are to collect their data and be able to control them. We re-engineered their functions and built services that: get current usage in Watts, total usage in kWh, switch their power supply on and off, get status, and some low-level information like calibration data, device info firmware, buffer data and internal clock.

Sensor Boards. Regardless the preconfigured sampling frequency of the sensors, services have been developed to always return temperature, humidity and luminance from a sensor along with timestamp of the reading, as well as averages, mimimum and maximum values for these readings in a given timespan.

Smart Clampers. Like Sensor Boards, Smart Clampers are sensors too, so data exposure suffices for integrating them in aWESoME. OWL Clamps are read through the company's API while CurrentCost Clampers dump data in XML. In both cases, services include getting consumption data, average, maximum and minimum values. 


\section{The iDEALISM Application}

The iDEALISM application (DEsktop AppLIcation for Smart Management) has been developed to fully exploit and showcase aWESoME's capabilities, which doesn't have a user-friendly UI, to preserve its generality. All in all, its novelty and energy saving potential lies in the fact that heterogeneous devices can be managed via the Web, and in the same place. The user can view live consumption and environmental data, and take informed decisions, even remotely. Also, subtracting plug consumption from clampers can help the user possibly locate an unwanted power waste. These are all functions that the bundles do not natively support.

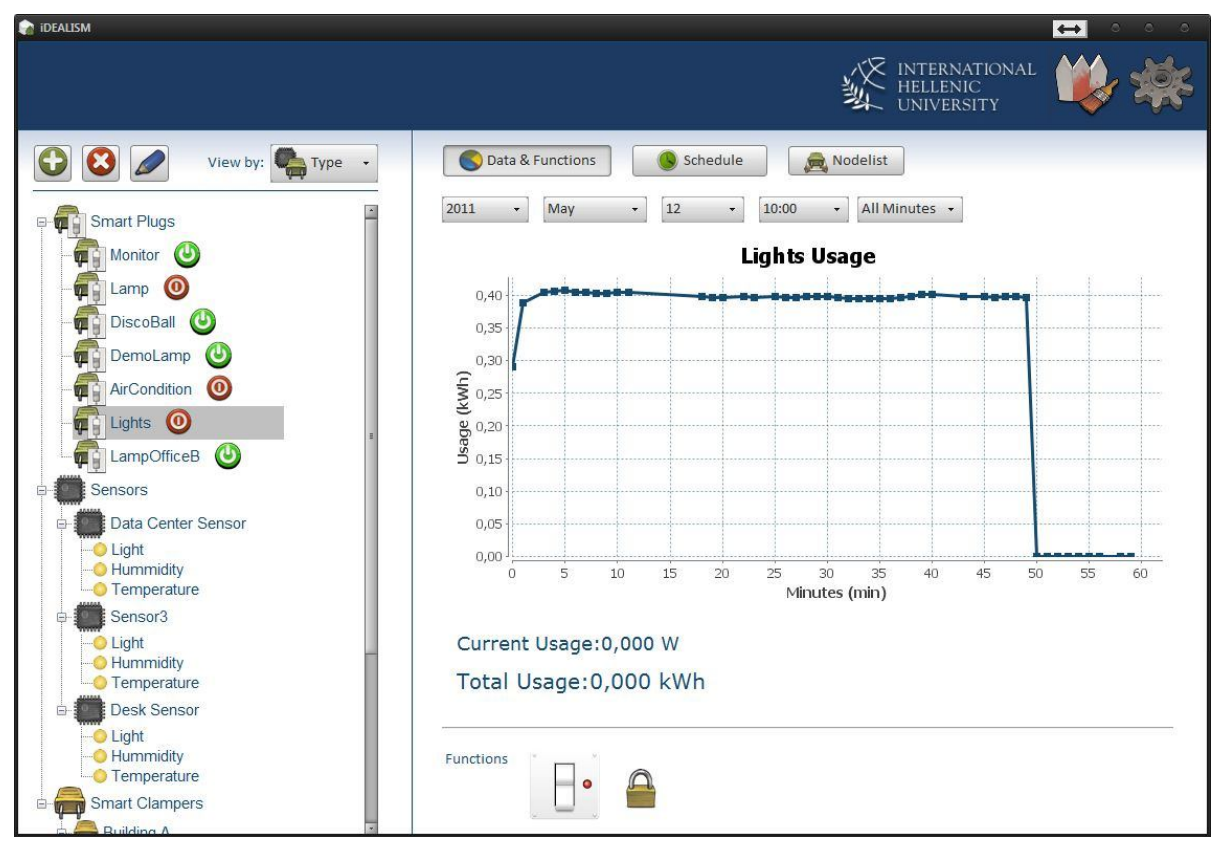

Fig. 2. iDEALISM GUI: The device tree is shown at the left pane and the corresponding chart and functions of the selected device at the right pane.

iDEALISM is shown on Figure 2. Nodes can be configured to operate locally or remotely, via Web Services. They can be viewed in an intuitive tree hierarchy per type, room or groups, so that even a large number of nodes can be managed easily. Data is stored in a local database and viewed in charts. Functions include switches, a handy lock and schedules. These can be extended in the future to add e.g. dimmer switches. There is also a status indicator for Smart Plugs. Finally, devices in the same group or room can be managed collectively.

User Evaluation. To evaluate the application's usability and effectiveness, thirty undergraduate computer science students tested it, having only little information about its function. In practice, users seem to quickly adapt to the interface and find what they were looking for. They completed different tasks and filled up a 
questionnaire, based on their experience. Table 2 shows some statistics in detail. Apparently, the application's look is very satisfying and testers consider the application to be effective in enabling energy save. Comments and suggestions on functionality didn't reveal any major missing functions. The on/off function, remote control over Web and schedules stood out as the most important functions for home use.

Table 2. Evaluation statistics: user satisfaction ratings from one to five

\begin{tabular}{lccc}
\hline Aspect & Average & Minimum & Maximum \\
\hline Overall Look & 4.3 & 3 & 5 \\
Layout usability & 4.1 & 3 & 5 \\
Amount of information/data & 4.1 & 3 & 5 \\
Functionality & 4.0 & 3 & 5 \\
Would you use it at home? & 4.2 & 2 & 5 \\
Would it make you save energy? & 4.3 & 2 & 5 \\
\hline
\end{tabular}

\section{Future Work}

Being the first deployment and implementation of this system, this work has much room for improvement. We plan to integrate many more sensors (e.g. CO2) and actuators, of Z-Wave or any other protocol, to enrich the variety and functions. Microcomputers are also investigated to replace PC gateways, to severely cut down the system's consumption. aWESoME and iDEALISM should support these new devices and present new ways to manage that data.

Then, a better method for service discovery should be utilized. The development of an ontology for the Web Service descriptions in this domain, would greatly contribute to this. Semantic annotations would render descriptions machine-interpretable and enable reasoning, automated service matching, and hence better discovery.

Finally, better matching would further facilitate automated composition using A.I. Planning. This is a well-known approach, as mentioned in section 2, that would provide the users with solutions to complex tasks and increase comfort of use of the AmI system.

\section{Conclusion}

This work presents both a middleware for AmI (aWESoME) and a client application (iDEALISM) that targets energy savings. Sets of devices like Smart Plugs, Sensor Boards and Smart Clampers have been deployed in large scale, in our University. aWESoME unifies these heterogeneous device sets and lifts all limitations regarding gateway placement and nodes per network. Devices can now be managed via the Web by a variety of desktop or web applications and Service Composition can be supported. iDEALISM is a desktop application of that kind, that breakthroughs in presenting and managing the sets of incompatible devices all in the same place. That 
allows comparisons and motivates users take informed decisions to save energy. All in all, there is much room for improvement by supporting more device classes and automating tasks.

Acknowledgments. This project is funded by Operational Program Education and Lifelong Learning, OPS 200056 (International Hellenic University, Thessaloniki, Greece).

\section{References}

1. Mark Weiser, The computer for the 21st century, ACM SIGMOBILE Mobile Computing and Communications Review, v.3 n.3, 3--11, (July 1999)

2. Thanos G. Stavropoulos, Ageliki Tsioliaridou, George Koutitas, Dimitris Vrakas, Ioannis P. Vlahavas: System Architecture for a Smart University Building. ICANN (3) 2010: 477-482

3. O. Davidyuk, N. Georgantas, V. Issarny, J. Riekki, MEDUSA: Middleware for End-User Composition of Ubiquitous Applications, in: Handbook of Research on Ambient Intelligence and Smart Environments: Trends and Perspectives, IGI Global, 2010

4. Ben Mokhtar, S. (2007). Semantic Middleware for Service-Oriented Pervasive Computing. Doctoral dissertation, University of Paris 6, Paris, France.K. Elissa, "Title of paper if known," unpublished.

5. Paluska, J. M., Pham, H., Saif, U., Chau, G., Terman, C. \& Ward, S. (2008). Structured decomposition of adaptive applications. International Journal of Pervasive and Mobile Computing, 4(6), 791--806

6. Brent Lagesse, Mohan Kumar, Matthew Wright: ReSCo: A middleware component for Reliable Service Composition in pervasive systems. PerCom Workshops 2010: 486-491

7. Wisner P. \& Kalofonos D.N. (2007). A Framework for End-User Programming of Smart Homes Using Mobile Devices. Proceedings of the 4th IEEE Consumer Communications and Networking Conference CCNC'07 (pp. 716--721), Washington DC: IEEE Computer Society.

8. Messer, A., Kunjithapatham, A., Sheshagiri, M., Song, H., Kumar, P., Nguyen, P. \& Yi, K.H. (2006). InterPlay: A Middleware for Seamless Device Integration and Task Orchestration in a Networked Home. Proceedings of the Annual IEEE International Conference on Pervasive Computing PerCom'06 (pp. 296--307), Washington DC: IEEE Computer Society.

9. André Bottaro, Anne Gérodolle, Home SOA - Facing Protocol Heterogeneity in pervasive Applications, 5th IEEE International Conference on Pervasive Services (ICPS 2008), Sorrento, Italy, July 2008

10. Mingkhwan, P. Fergus, O. Abuelma'Atti, M. Merabti, B. Askwith, and M. B. Hanneghan. Dynamic service composition in home appliance networks. Multimedia Tools and Applications, 29(3):257--284, 2006

11. Hydra Middleware, http://www.hydramiddleware.eu

12. The ZigBee Alliance, http://www.zigbee.org/

13. X10, http://kbase.x10.com/

14. INSTEON, http://www.insteon.net/

15. The Z-Wave Alliance, http://www.Z-wavealliance.org/

16. Plugwise, http://www.plugwise.com/

17. Prisma Electronics, http://www.prismaelectronics.eu/

18. 2SE - OWL, http://www.theowl.com/

19. CurrentCost, http://www.currentcost.com/ 